\title{
The Impacts of Albuminuria and eGFR on Cardiovascular Disease
}

\author{
Hao-Huan Hu ${ }^{1}$, Chin-Wen Hsieh ${ }^{1}$, Yu-Kuei Liao ${ }^{2}$, Szu-Mei Hsiao ${ }^{3}$, Pi-Li Lin ${ }^{3}$, Aih-Fung Chiu ${ }^{3}$, \\ Tsan Yang, ${ }^{*}$ * \\ ${ }^{1}$ Division of Nephrology, Pingtung Christian Hospital, Pingtung, Taiwan \\ ${ }^{2}$ Department of Nursing, Pingtung Christian Hospital, Pingtung, Taiwan \\ ${ }^{3}$ Department of Nursing, Meiho University, Pingtung, Taiwan \\ ${ }^{4}$ Department of Health Business Administration, Meiho University, Pingtung, Taiwan
}

Email address:

tsan.yang@msa.hinet.net (Tsan Yang)

${ }^{*}$ Corresponding author

\section{To cite this article:}

Hao-Huan Hu, Chin-Wen Hsieh, Yu-Kuei Liao, Szu-Mei Hsiao, Pi-Li Lin, Aih-Fung Chiu, Tsan Yang. The Impacts of Albuminuria and eGFR on Cardiovascular Disease. American Journal of Health Research. Vol. 5, No. 4, 2017, pp. 99-105. doi: 10.11648/j.ajhr.20170504.12

Received: May 14, 2017; Accepted: May 25, 2017; Published: July 11, 2017

\begin{abstract}
Albuminuria is often used as a surrogate marker for the risk of fatal and non-fatal events in clinical trials of antihyperglycemic medications or in antihypertensive therapy. Similarly, low estimated glomerular filtration rate (eGFR), which is a common manifestation of progressed diabetic nephropathy, has also been demonstrated to be an independent risk factor for cardiovascular events and death. Recent evidence suggests that both high albuminuria and low eGFR are independent risk factors for progressive kidney failure and cardiovascular disease. The purpose of this study was to investigate the impacts of albuminuria and low eGFR on the risk of cardiovascular disease. A cross-sectional design was used. Data were collected through adults' health examinations by a hospital in a certain area in Pingtung County between 2011 and 2015. The health data base included participants' basic information, physical examination and blood examination results. Use abbreviated modification of diet in renal disease, aMDRD (Abbreviated modification of diet in renal disease) formula to estimate eGFR. Use metabolic syndrome to define the criteria of rising blood pressure, blood sugar, blood lipids as an important cardiovascular disease (CVD) indicator and then calculation of the 10-year risk for CVD was completed using data from the Framingham Heart Study and a computer was used to determine risk values. In this study, $\leq 10 \%$ was defined as low risk, $11-20 \%$ was defined as moderate risk, and $>20 \%$ was defined as high risk. As albuminuria and eGFR approached critically high values, initially moderate and high 10 -year risk levels for CVD tended to increase. Logistic regression analysis showed that patients with severe albuminuria and severe eGFR had higher risks of metabolic syndrome, abnormal waist circumference, hyperglycemia, reduced high-density lipoprotein cholesterol (HDL-C), hypertriglyceridemia, and elevated blood pressure. The study concluded that albuminuria and eGFR are risk factors for CVD and can increase a patient's 10-year risk of CVD.
\end{abstract}

Keywords: Albuminuria, Glomerular Filtration Rate, Cardiovascular Disease, Metabolic Syndrome

\section{Introduction}

Chronic kidney disease (CKD) is an important public health issue on the global stage $[1,2] .10-16 \%$ of adults in Asia, Australia, Europe, and America suffer from CKD [3-6]. Even after the exclusion of traditional CKD risk factors, such as hypertension and diabetes, $\mathrm{CKD}$ has been found to increase all-cause death (ACD), mortality due to cardiovascular diseases (CVD), and the risk of death due to renal failure [1, 7].
Many studies have indicated that estimated glomerular filtration rate (eGFR) and albuminuria are related to CKD in the general population. One study found that lower eGFR $(<$ $\left.60 \mathrm{ml} / \mathrm{min} / 1.73 \mathrm{~m}^{2}\right)$ and higher albuminuria (albumin-to-creatinine ratio; ACR $\geq 10 \mathrm{mg} / \mathrm{g}$ ) levels are independent risk factors for ACD and death due to CVD in the general population [8]. 
CKD is characterized by decreased renal function (GFR < $60 \mathrm{ml} / \mathrm{min} / 1.73 \mathrm{~m}^{2}$ ) or renal injuries (by urinary albumin excretion $\geq 30 \mathrm{mg} /$ day) [9]. GFR is used to diagnose CKD [2, $10]$ and is widely applied as an independent factor to predict $\mathrm{ACD}$, death due to $\mathrm{CVD}$, and renal failure in the general population $[8,11-13]$. For the measurement of serum creatinine, clinical guidelines recommend using eGFR $[2,10]$ as the renal function indicator. Currently, eGFR is used in $84 \%$ of laboratories in the United States [14]. eGFR and albuminuria are important factors for CKD diagnosis, staging, and risk assessment. For universal thresholds, the use of this standard is recommended regardless of age, gender, and ethnic group. However, the relation between demographic factors and the impacts of eGFR and albuminuria on CVD is understudied [15].

Recent studies have shown that albuminuria and low eGFR are independent risk factors for renal failure and CVD. Nevertheless, different research designs and different methods of participant selection may lead to different results regarding renal failure, CVD, and ACD.

There have been few studies in Taiwan that evaluated the effects of albuminuria and low eGFR on CVD risk. This study investigated the impacts of albuminuria and eGFR on CVD.

\section{Methods}

\subsection{Study Design}

This study conducted a cross-sectional research investigation. Data were collected from health examinations for adults conducted by a hospital in Pingtung County between 2011 and 2015. Data obtained from the health database (including the participants' basic information, as well as physical examination and blood and urinary examination results) were processed. The abbreviated modification of diet in renal disease (aMDRD) formula was used to estimate eGFR. Metabolic syndrome and compositional factors served as important criteria for CVD. The 10-year risk for CVD was calculated using the assessment tool from the Framingham Heart Study; $\leq 10 \%$ was defined as low risk, $11-20 \%$ was defined as moderate risk, and $>20 \%$ was defined as high risk.

\subsection{Participants}

The participants in this study were adults who underwent health examinations (or comprehensive screening) between 2011 and 2015; those who did not complete their physical examinations or biochemical blood tests were excluded.

\subsection{Definition of Terms}

\subsubsection{Definition of CKD}

In the present study, the GFR was estimated using the Modification of Diet in Renal Disease (MDRD), and CKD was grouped into 5 stages based on the categorization of CKD by the National Kidney Foundation, Inc.: a patient whose eGFR $\geq 90 \mathrm{ml} / \mathrm{min} / 1.73 \mathrm{~m}^{2}$ with proteinuria was in stage 1 ; those with eGFR $60-89 \mathrm{ml} / \mathrm{min} / 1.73 \mathrm{~m}^{2}$ with proteinuria were in stage 2 ; those with eGFR $30-59 \mathrm{ml} / \mathrm{min} / 1.73 \mathrm{~m}^{2}$ were in stage 3 ; those with eGFR $15-29 \mathrm{ml} / \mathrm{min} / 1.73 \mathrm{~m}^{2}$ were in stage 4 ; those with eGFR $<15 \mathrm{ml} / \mathrm{min} / 1.73 \mathrm{~m}^{2}$ were in stage 5 .

\subsubsection{Metabolic Syndrome Was Defined According to the Criteria Set by the Health Promotion Administration, Ministry of Health and Welfare, in 2007}

Accordingly, three of the following five criteria were grounds for definition: (1) elevated blood pressure: blood pressure of at least $130 / 85 \mathrm{mmHg}$ or use of antihypertensive medications, (2) hypertriglyceridemia: serum triglycerides (TG) of at least $150 \mathrm{mg} / \mathrm{dL}$, (3) reduced high-density lipoprotein cholesterol (HDL-C): HDL-C $<40 \mathrm{mg} / \mathrm{dL}$ in men and $<50 \mathrm{mg} / \mathrm{dL}$ in women, (4) hyperglycemia: raised fasting plasma glucose (FPG) of $100 \mathrm{mg} / \mathrm{dL}$ or more or use of drug treatment of elevated glucose, and (5) central obesity: waist circumference $\geq 90 \mathrm{~cm}$ in men and $\geq 80 \mathrm{~cm}$ in women.

\subsubsection{High-Risk Factors of CVD}

Metabolic syndrome and compositional factors (elevated blood pressure, hyperglycemia, hypertriglyceridemia, reduced HDL-C, and central obesity) were important criteria for CVD.

\subsubsection{A Qualitative Examination Was Conducted to Determine Albuminuria Levels}

A reagent paper was placed into urine. Results were categorized into: negative (-) (albumin $<0.1 \mathrm{~g} / \mathrm{L})$, trace $(+/-)$ $(0.1-0.2 \mathrm{~g} / \mathrm{L}), 1+(0.2-1.0 \mathrm{~g} / \mathrm{L}), 2+(1.0-2.0 \mathrm{~g} / \mathrm{L}), 3+(2.0-4.0 \mathrm{~g} / \mathrm{L})$, and $4+(>4.0 \mathrm{~g} / \mathrm{L})$.

\subsubsection{The 10-Year CVD Risk Assessment Tool from the Framingham Heart Study Was Employed to Calculate the Risk}

Data from the Framingham Heart Study were used to predict the chances of CVD in the following 10 years. $\leq 10 \%$ was defined as low risk, $11-20 \%$ was defined as moderate risk, and $>20 \%$ was defined as high risk. [16]

\subsection{Ethical Considerations}

The data collection and analysis in this study began after the research plan was reviewed by the Institutional Review Board (IRB).

\subsection{Statistical Analysis}

Statistics Package for Social Science (SPSS) 18.0 software was used for data analysis. Inferential statistics applied the Chi-Square test $(\chi 2)$, multinomial logistic regression, and logistic regression.

\section{Results}

With regard to Table 1, the 10-year CVD risk assessment tool from the Framingham Heart Study was employed in this study to calculate risk. Data from the Framingham Heart Study was used to predict the probability of CVD developing in the next 10 years of a person's life. $\leq 10 \%$ was defined as low risk, $11-20 \%$ was defined as moderate risk, and $>20 \%$ 
was defined as high risk. The effects of eGFR staging and albuminuria levels on CVD risk were analyzed. The results indicated that as abnormally high levels of eGFR and albuminuria increased, so did the risk of CVD; the correlation was significant in both cases.

Table 1. Impact of eGFR and albuminuria on 10-year risk of CVD $(n=8850)$.

\begin{tabular}{|c|c|c|c|c|c|c|c|}
\hline \multirow[b]{2}{*}{ Variable } & \multicolumn{2}{|l|}{$\leq 10(n=5318)$} & \multicolumn{2}{|c|}{$11-20(n=2390)$} & \multicolumn{2}{|l|}{$>20(n=1142)$} & \multirow[b]{2}{*}{ *p value } \\
\hline & Number of people & Percentage & $\begin{array}{l}\text { Number of } \\
\text { people }\end{array}$ & Percentage & $\begin{array}{l}\text { Number of } \\
\text { people }\end{array}$ & Percentage & \\
\hline eGFR staging & & & & & & & $<.001$ \\
\hline 0 & 4132 & 68.6 & 1421 & 23.6 & 471 & 7.8 & \\
\hline 1 & 484 & 68.2 & 170 & 23.9 & 56 & 7.9 & \\
\hline 2 & 441 & 41.4 & 398 & 37.4 & 226 & 21.2 & \\
\hline 3 & 215 & 23.5 & 350 & 38.3 & 349 & 38.2 & \\
\hline 4 & 32 & 33.0 & 36 & 37.1 & 29 & 29.9 & \\
\hline 5 & 14 & 35.0 & 15 & 37.5 & 11 & 27.5 & \\
\hline eGFR staging & & & & & & & $<.001$ \\
\hline 0 & 4132 & 68.6 & 1421 & 23.6 & 471 & 7.8 & \\
\hline 1 & 925 & 52.1 & 568 & 32.0 & 282 & 15.9 & \\
\hline 2 & 261 & 24.8 & 401 & 38.2 & 389 & 37.0 & \\
\hline Albuminuria & & & & & & & $<.001$ \\
\hline- & 3508 & 65.3 & 1335 & 24.8 & 533 & 9.9 & \\
\hline$-/+$ & 1351 & 56.5 & 706 & 29.5 & 335 & 14.0 & \\
\hline $1+$ & 375 & 44.8 & 274 & 32.7 & 188 & 22.5 & \\
\hline $2+$ & 59 & 35.3 & 50 & 29.9 & 58 & 34.7 & \\
\hline 3 and higher & 25 & 32.1 & 25 & 32.1 & 28 & 35.9 & \\
\hline Albuminuria & & & & & & & $<.001$ \\
\hline- & 3508 & 65.3 & 1335 & 24.8 & 533 & 9.9 & \\
\hline$-/+$ & 1351 & 56.5 & 706 & 29.5 & 335 & 14.0 & \\
\hline $1+$ & 375 & 44.8 & 274 & 32.7 & 188 & 22.5 & \\
\hline 2 and higher & 84 & 34.3 & 75 & 30.6 & 86 & 35.1 & \\
\hline
\end{tabular}

${ }^{\#}$ Stage of eGFR: 0(non CKD); 1(eGFR $\geq 90 \mathrm{ml} / \mathrm{min} / 1.73 \mathrm{~m}^{2}$ with proteinuria); 2(eGFR 60-89 $\mathrm{ml} / \mathrm{min} / 1.73 \mathrm{~m}^{2}$ with $\left.\mathrm{proteinuria}\right) ; 3\left(\mathrm{eGFR} 30-59 \mathrm{ml} / \mathrm{min}^{2} 1.73 \mathrm{~m}^{2}\right)$; $4\left(\mathrm{eGFR} 15-29 \mathrm{ml} / \mathrm{min} / 1.73 \mathrm{~m}^{2}\right) ; 5\left(\mathrm{eGFR}<15 \mathrm{ml} / \mathrm{min} / 1.73 \mathrm{~m}^{2}\right)$

\#\# Stage of eGFR: 0(non CKD); 1 (eGFR $\geq 60 \mathrm{ml} / \mathrm{min} / 1.73 \mathrm{~m}^{2}$ with proteinuria ); $2\left(\mathrm{eGFR}<60 \mathrm{ml} / \mathrm{min} / 1.73 \mathrm{~m}^{2}\right)$

*The Chi-square (two-tailed) test was used to compare the variables, with a significance level of $\alpha=.05$.

Table 2 shows the effects of albuminuria and eGFR on CVD risk $(\leq 10 \% ; 11-20 \% ;>20 \%)$ based on a multinomial logistic regression analysis. The results indicated that as albuminuria and eGFR approached critically high values, initially moderate and high 10-year risk levels for CVD tended to increase. In the case of patients with a moderate 10 -year risk of CVD, the odds ratios (ORs) in patients with $+/-, 1+$, and $\geq$ $2+$ albuminuria were $1.18,1.26$, and 1.59 , respectively. The
ORs in patients with eGFR $\geq 60 \mathrm{ml} / \mathrm{min} / 1.73 \mathrm{~m}^{2}$ with proteinuria and eGFR $<60 \mathrm{ml} / \mathrm{min} / 1.73 \mathrm{~m}^{2}$ were 1.10 and 2.36 , respectively. In the case of patients with a high 10 -year risk of CVD, the ORs in patients with $+/-, 1+$, and $\geq 2+$ albuminuria were $1.16,1.56$, and 3.39 , respectively. The ORs in patients with $\mathrm{eGFR} \geq 60 \mathrm{ml} / \mathrm{min} / 1.73 \mathrm{~m}^{2}$ with proteinuria and eGFR < $60 \mathrm{ml} / \mathrm{min} / 1.73 \mathrm{~m}^{2}$ were 1.54 and 5.35 , respectively.

Table 2. 10-year risk factors of CVD.

\begin{tabular}{|c|c|c|c|c|}
\hline \multirow{2}{*}{ Variable } & \multirow{2}{*}{$\begin{array}{l}\text { Moderate risk } \\
\text { OR }^{\#}(95 \% \text { CI) } \\
\end{array}$} & \multirow{2}{*}{$p$ value } & \multirow{2}{*}{$\begin{array}{l}\text { High risk } \\
\text { OR }(95 \% \text { CI }) \\
\end{array}$} & \multirow{2}{*}{$p$ value } \\
\hline & & & & \\
\hline Gender & $51.19(41.76-62.73)$ & $<.001$ & 219.00(159.24-301.18) & $<.001$ \\
\hline Age & $22.12(18.22-26.84)$ & $<.001$ & $127.23(95.93-168.75)$ & $<.001$ \\
\hline Albuminuria +/- & $1.18(0.96-1.44)$ & 0.112 & $1.16(0.87-1.54)$ & 0.310 \\
\hline Albuminuria 1+ & $1.26(0.98-1.63)$ & 0.076 & $1.56(1.12-2.19)$ & 0.010 \\
\hline Albuminuria $2+$ and higher & $1.59(1.06-2.38)$ & 0.026 & $3.39(2.08-5.51)$ & $<.001$ \\
\hline eGFR1 & $1.10(0.88-1.37)$ & 0.412 & $1.54(1.13-2.11)$ & 0.006 \\
\hline eGFR2 & $2.36(1.86-2.98)$ & $<.001$ & $5.35(4.01-7.14)$ & $<.001$ \\
\hline
\end{tabular}

In Table 3, metabolic syndrome and compositional factors (central obesity, elevated blood pressure, hyperglycemia, hypertriglyceridemia, and reduced HDL-C) are listed as dependent variables that are considered to be risk factors for CVD. Logistic regression analysis was used to examine the effects of albuminuria and eGFR on these variables. The results indicated that at more critical levels of albuminuria and eGFR, there was a higher risk of metabolic syndrome and abnormalities related to compositional factors. 
Table 3. Predictive analysis of the effects on CVD risk factors.

\begin{tabular}{|c|c|c|c|c|}
\hline Regression model & $\beta$ & wald & OR(95\%CI) & *p value \\
\hline \multicolumn{5}{|c|}{ Metabolic syndrome (yes vs. no) } \\
\hline Gender & -0.17 & 13.07 & $0.85(0.77-0.93)$ & $<.001$ \\
\hline Age & 0.49 & 107.37 & $1.63(1.48-1.78)$ & $<.001$ \\
\hline Albuminuria $+/-$ & 0.24 & 21.90 & $1.28(1.15-1.41)$ & $<.001$ \\
\hline Albuminuria 1+ & 0.65 & 69.29 & $1.91(1.64-2.22)$ & $<.001$ \\
\hline Albuminuria $2+$ and higher & 1.34 & 76.87 & $3.80(2.82-5.12)$ & $<.001$ \\
\hline eGFR2 & 0.52 & 50.64 & $1.69(1.46-1.95)$ & $<.001$ \\
\hline \multicolumn{5}{|c|}{ Waist circumference (abnormal vs. normal) } \\
\hline Gender & -0.48 & 115.39 & $0.62(0.57-0.67)$ & $<.001$ \\
\hline Age & 0.47 & 103.08 & $1.59(1.45-1.74)$ & $<.001$ \\
\hline Albuminuria $1+$ & 0.20 & 6.85 & $1.23(1.05-1.43)$ & 0.009 \\
\hline Albuminuria $2+$ and higher & 0.64 & 19.9 & $1.90(1.43-2.52)$ & $<.001$ \\
\hline eGFR1 & 0.19 & 11.11 & $1.21(1.08-1.36)$ & 0.001 \\
\hline eGFR2 & 0.41 & 29.33 & $1.51(1.30-1.75)$ & $<.001$ \\
\hline \multicolumn{5}{|c|}{ Hperglycemia (abnormal vs. normal) } \\
\hline Gender & 0.18 & 15.63 & $1.19(1.09-1.30)$ & $<.001$ \\
\hline Age & 0.52 & 132.05 & $1.69(1.55-1.85)$ & $<.001$ \\
\hline Albuminuria +/- & 0.22 & 19.77 & $1.25(1.13-1.38)$ & $<.001$ \\
\hline Albuminuria $1+$ & 0.71 & 81.42 & $2.02(1.74-2.36)$ & $<.001$ \\
\hline Albuminuria $2+$ and higher & 1.23 & 63.14 & $3.42(2.53-4.64)$ & $<.001$ \\
\hline eGFR2 & 0.26 & 12.78 & $1.30(1.13-1.51)$ & $<.001$ \\
\hline \multicolumn{5}{|c|}{ reduced HDL-C (abnormal vs. normal) } \\
\hline Age & 0.54 & 141.11 & $1.72(1.57-1.88)$ & $<.001$ \\
\hline Albuminuria +/- & 0.24 & 22.87 & $1.27(1.15-1.40)$ & $<.001$ \\
\hline Albuminuria 1+ & 0.73 & 87.27 & $2.07(1.78-2.41)$ & $<.001$ \\
\hline Albuminuria $2+$ and higher & 1.24 & 64.16 & $3.46(2.55-4.69)$ & $<.001$ \\
\hline eGFR2 & 0.29 & 15.32 & $1.33(1.16-1.54)$ & $<.001$ \\
\hline \multicolumn{5}{|c|}{ Hpertriglyceridemia (abnormal vs. normal) } \\
\hline Gender & 0.28 & 30.35 & $1.32(1.19-1.45)$ & $<.001$ \\
\hline Age & -0.23 & 20.49 & $0.79(0.72-0.88)$ & $<.001$ \\
\hline Albuminuria $2+$ and higher & 0.50 & 12.94 & $1.65(1.26-2.17)$ & $<.001$ \\
\hline eGFR1 & 0.20 & 10.35 & $1.22(1.08-1.37)$ & 0.001 \\
\hline eGFR2 & 0.37 & 21.27 & $1.45(1.24-1.70)$ & $<.001$ \\
\hline \multicolumn{5}{|c|}{ Elevated blood pressure (abnormal vs. normal) } \\
\hline Gender & 0.20 & 17.61 & $1.22(1.11-1.34)$ & $<.001$ \\
\hline Age & 1.23 & 607.02 & $3.41(3.09-3.76)$ & $<.001$ \\
\hline Albuminuria +/- & 0.11 & 3.90 & $1.11(1.00-1.23)$ & 0.048 \\
\hline Albuminuria $1+$ & 0.61 & 44.19 & $1.84(1.54-2.21)$ & $<.001$ \\
\hline Albuminuria $2+$ and higher & 1.26 & 33.11 & $3.54(2.30-5.44)$ & $<.001$ \\
\hline eGFR2 & 0.65 & 44.39 & $1.91(1.58-2.31)$ & $<.001$ \\
\hline
\end{tabular}

* Stepwise regression analysis was used. The following variables were included in the regression model: Gender (female), age ( $<65$ years old), albuminuria (-), and eGFR staging $(0)()$ is indicated as the reference group.

\section{Discussion}

Early albuminuria detection should be included in general screening to detect kidney abnormalities early. The levels of albuminuria are linearly correlated with nephropathy risk. Past research findings indicated that microalbuminuria is an indicator of diabetes complications and an independent risk factor for CVD. The Framingham Heart Study's 10-year CVD risk assessment tool was employed in this study to calculate the risk of CVD in the following 10 years. The results showed that 10-year CVD risk was significantly higher in patients with critically high levels of eGFR and albuminuria. Many studies on patients with Type 1 and 2 diabetes mellitus (DM) and albuminuria have indicated higher mortality rates due to CVD [17], findings which correspond to the results in this study.

One comprehensive meta-analysis report regarding research on Type 2 DM patients analyzed multiple CVD risk factors; the results revealed that after the exclusion of common risk factors, such as blood pressure and dyslipidemia, the CVD incidence and death risk in patients with microalbuminuria was two-fold higher than that in other patients. Therefore, microalbuminuria is an early indicator of diabetic renal lesions and a predictor of mortality due to CVD. The prevalence of microalbuminuria in non-diabetic patients with elevated blood pressure ranged between $5-40 \%$; in such patients, a correlation between microalbuminuria and mortality due to CVD can still be observed [18], which corresponds to the results in this study.

The analysis results from this study demonstrated that, as albuminuria and eGFR approached critical values, initially moderate and high 10-year risk levels for CVD tended to increase. Related studies have indicated that, in addition to its link to chronic kidney diseases, albuminuria is also an 
indicator of the risk of CVD and death due to CVD [19]. In this study, CVD risk factors (metabolic syndrome, central obesity, elevated blood pressure, hyperglycemia, hypertriglyceridemia, and reduced HDL-C) were set as dependent variables, and the impact of albuminuria and eGFR on those CVD risk factors were analyzed. The results indicated that patients with critical higher levels of albuminuria and eGFR have higher risks of abnormality in CVD risk factors. However, few studies have examined the effects of albuminuria and eGFR on metabolic syndrome and compositional factors set as CVD risk factors. The findings of this study were compared only with the results reported by related studies and were found to have been consistent with them. A study on young and middle-aged non-diabetic and without hypertension male workers found a relation between high low-density lipoprotein cholesterol (LDL-C) and CKD incidence and reduced eGFR [20]. A study on Japanese participants indicated the influence of a higher TG/HDL-C ratio on a decrease in eGFR and the aggravation of CKD [21]. Another one-year short-term study revealed a significant correlation between low HDL-C and a decrease in eGFR. Metabolic syndrome also showed a significant association with eGFR decline. A study of a non-CKD population found that hypertension and low HDL-C associated with metabolic syndrome had a greater effect on eGFR than obesity [22]. A study involving a healthy Korean population revealed that metabolic syndrome and insulin resistance are independent risk factors for incident $\mathrm{CKD}$ and a rapid decline of eGFR [23]. Some studies also found that insulin resistance is related to $\mathrm{CKD}$ incidence and the decline of renal function in older adults [24]. Low eGFR is significantly and independently correlated with 6-month functional outcomes and mortality in patients with the large artery atherosclerotic (LAA) subtype of acute ischemic stroke [25]. Furthermore, a study of stroke patients found that low eGFR can increase the risk of ACD and recurrent stroke, independent of traditional CVD risk factors [26].

Other studies have found that the effects of eGFR and albumin-to-creatinine ratio (ACR) on cardiovascular events were largely similar. $\mathrm{CKD}$ is also a common cause of increased CVD risk [8]. One follow-up study on the high risks of ACD and CVD in middle-aged and older adults indicated that the simultaneous presence of eGFR and albuminuria can increase the risk of ACD, cardiovascular death, and neoplasm-related death [27]. Past studies also found that a moderate to severe decline of GFR is related to high risks of CVD incidence and death [28-30]. Recent studies have indicated a U-shaped relationship between estimated GFR (eGFR) and the risk of all-cause death [8, 31-34]. According to Tonelli et al., the results for normal and higher eGFR levels with or without albuminuria are similar to those for reduced eGFR levels without albuminuria, with both causing high risks of death [31, 35], findings which correspond to the results in this study. Other studies have found that the decline of eGFR is a risk factor for CVD aggravation, including acute coronary syndrome [36], cardiac failure [37, 38], and acute myocardial infarction [39]. However, the cause-effect relationship between renal function support and CVD and its mechanism has yet to be determined [40, 41].

The study still had some limitations that are worth noting. First, data was derived only from one hospital; as such, due to sample deviation, the results may not be generalizable to all hospitals in Taiwan. However, as a large sample was used, the results may be used by related studies as a reference. Second, this study only investigated the correlations of demographic data, physical examination data, and biochemical blood test data to metabolic syndrome and CVD risk factors. Moreover, only examination data was analyzed and not all potential influencing factors of CVD were included. Therefore, inferences must be made carefully.

\section{Conclusion}

We concluded that albuminuria and eGFR approached critically high values, there are higher risks for abnormalities in metabolic syndrome, abnormal waist circumference, hyperglycemia, reduced high-density lipoprotein cholesterol (HDL-C), hypertriglyceridemia, and elevated blood pressure. Albuminuria and eGFR are risk factors for CVD and can increase a patient's 10-year risk of CVD.

\section{References}

[1] Levey AS, Atkins R, Coresh J, et al. Chronic kidney disease as a global public health problem: approaches and initiatives - a position statement from Kidney Disease Improving Global Outcomes. Kidney Int. 2007; 72: 247-59. [PubMed: 17568785].

[2] National Kidney Foundation. K/DOQI clinical practice guidelines for chronic kidney disease: evaluation, classification, and stratification. Am J Kidney Dis. 2002; 39: S1-266. [PubMed: 11904577].

[3] Wen CP, Cheng TY, Tsai MK, et al. All-cause mortality attributable to chronic kidney disease: a prospective cohort study based on 462293 adults in Taiwan. Lancet. 2008; 371: 2173-82. [PubMed: 18586172].

[4] Chadban SJ, Briganti EM, Kerr PG, et al. Prevalence of kidney damage in Australian adults: The AusDiab kidney study. J Am Soc Nephrol. 2003; 14: S131-8. [PubMed: 12819318].

[5] Hallan SI, Coresh J, Astor BC, et al. International comparison of the relationship of chronic kidney disease prevalence and ESRD risk. J Am Soc Nephrol. 2006; 17: 2275-84. [PubMed: 16790511].

[6] Coresh J, Selvin E, Stevens LA, et al. Prevalence of chronic kidney disease in the United States. JAMA. 2007; 298: 2038 47. [PubMed: 17986697].

[7] Sarnak MJ, Levey AS, Schoolwerth AC, et al. Kidney disease as a risk factor for development of cardiovascular disease: a statement from the American Heart Association Councils on Kidney in Cardiovascular Disease, High Blood Pressure Research, Clinical Cardiology, and Epidemiology and Prevention. Circulation. 2003; 108: 2154-69. [PubMed: 14581387]. 
[8] Matsushita K, van der Velde M, Astor BC, et al. Association of estimated glomerular filtration rate and albuminuria with all-cause and cardiovascular mortality in general population cohorts: a collaborative meta-analysis. Lancet. 2010; 375(9731): 2073-2081. [PubMed: 20483451].

[9] Kidney Disease: Improving Global Outcomes (KDIGO) CKD Work Group. KDIGO 2012 Clinical Practice Guideline for the Evaluation and Management of Chronic Kidney Disease. Kidney Int. 2013; (Suppl. 3) (1): 1-150.

[10] Crowe E, Halpin D, Stevens P, on behalf of the Guideline Development G. Early identification and management of chronic kidney disease: summary of NICE guidance. BMJ. 2008; 337(sep29 1): a1530. [PubMed: 18824486].

[11] van der Velde M, Matsushita K, Coresh J, et al. Lower estimated glomerular filtration rate and higher albuminuria are associated with all-cause and cardiovascular mortality. A collaborative metaanalysis of high-risk population cohorts. Kidney Int. 2011; 79(12): 1341-1352. [PubMed: 21307840].

[12] Gansevoort RT, Matsushita K, van der Velde M, et al. Lower estimated GFR and higher albuminuria are associated with adverse kidney outcomes. A collaborative meta-analysis of general and high-risk population cohorts. Kidney Int. 2011; 80(1): 93-104. [PubMed: 21289597].

[13] Astor BC, Matsushita K, Gansevoort RT, et al. Lower estimated glomerular filtration rate and higher albuminuria are associated with mortality and end-stage renal disease. A collaborative meta-analysis of kidney disease population cohorts. Kidney Int. 2011; 79(12): 1331-1340. [PubMed: 21289598].

[14] The College of American Pathologists. [Accessed March, 6, 2012] Current Status Of Reporting Estimated Glomerular Filtration Rate (eGFR) for Adults. http://www.cap.org/apps/docs/committees/ chemistry/current_status_of_reporting_eGF R_2011.pdf.

[15] Hui X, Matsushita K, Sang Y, et al. CKD and cardiovascular disease in the Atherosclerosis Risk in Communities (ARIC) study: interactions with age, sex, and race. Am J Kidney Dis. 2013 Oct; 62(4): 691-702. doi: 10.1053/j.ajkd.2013.04.010. Epub 2013 Jun 13.

[16] Expert Panel on Detection, Evaluation, and Treatment of High Blood Cholesterol in Adults. Executive Summary of the Third Report of The National Cholesterol Education Program (NCEP) Expert Panel on Detection, Evaluation, And Treatment of High Blood Cholesterol In Adults (Adult Treatment Panel III). JAMA. 2001 May, 285(19): 2486-2497.

[17] Lee PH, Chang HY, Tung CW, et al. Microalbuminuria, a Sign Usually Ignored; Journal of Internal Medicine of Taiwan. 2009; 20(4), 275 - 284. DOI: 10.6314/JIMT.2009.20(4).01.

[18] Garg JP, Bakris GL. Microalbuminuria: marker of vascular dysfunction, risk factor for cardiovascular disease. Vasc Med 2002; 7: 35-43.

[19] Hillege H, Van Gilst W, de Zeeuw D, et al. Renal function as a predictor of prognosis in chronic heart failure. Heart Fail Monit. 2002; 2(3): 78-84.

[20] Kuma A, Uchino B, Ochiai Y, et al. Impact of low-density lipoprotein cholesterol on decline in estimated glomerular filtration rate in apparently healthy young to middle-aged working men. Clin Exp Nephrol. 2017 Apr 6. doi: 10.1007/s10157-017-1407-8. PMID: 28386655.
[21] Tsuruya $\mathrm{K}$, Yoshida $\mathrm{H}$, Nagata $\mathrm{M}$, et al. Impact of the Triglycerides to High-Density Lipoprotein Cholesterol Ratio on the Incidence and Progression of CKD: A Longitudinal Study in a Large Japanese Population. Am J Kidney Dis. 2015 Dec; 66(6): 972-83. doi: 10.1053/j.ajkd.2015.05.011. PMID: 26145254.

[22] Hayashi K, Takayama M, Abe T, et al. Investigation of Metabolic Factors Associated with eGFR Decline Over 1 Year in a Japanese Population without CKD. J Atheroscler Thromb. 2017 Jan 26. doi: 10.5551/jat.38612. PMID: 28123142.

[23] Huh JH, Yadav D, Kim JS, et al. An association of metabolic syndrome and chronic kidney disease from a 10-year prospective cohort study. Metabolism. $2017 \mathrm{Feb;} \mathrm{67:} \mathrm{54-61.}$ doi: 10.1016/j.metabol.2016.11.003. PMID: 28081778.

[24] Cheng HT, Huang JW, Chiang CK, et al. Metabolic syndrome and insulin resistance as risk factors for development of chronic kidney disease and rapid decline in renal function in elderly. J Clin Endocrinol Metab. 2012 Apr; 97(4): 1268-76. doi: 10.1210/jc.2011-2658. PMID: 22337909.

[25] Yeh SJ, Jeng JS, Tang SC, et al. Low estimated glomerular filtration rate is associated with poor outcomes in patients who suffered a large artery atherosclerosis stroke. Atherosclerosis. 2015 Apr; 239(2): 328-34. doi: 10.1016/j.atherosclerosis.2015.01.038. PMID: 25682031.

[26] Wang X, Wang Y, Wang C, et al. Association between estimated glomerular filtration rate and clinical outcomes in patients with acute ischaemic stroke: results from China National Stroke Registry. Age Ageing. 2014 Nov; 43(6): 839-45. doi: 10.1093/ageing/afu090. PMID: 25141853.

[27] Ohsawa M, Fujioka T, Ogasawara K, et al. High risks of all-cause and cardiovascular deaths in apparently healthy middle-aged people with preserved glomerular filtration rate and albuminuria: A prospective cohort study. Int J Cardiol. 2013 Dec 10; 170(2): 167-72. doi: 10.1016/j.ijcard.2013.10.076. Epub 2013 Oct 29.

[28] Go AS, Chertow GM, Fan D, et al. Chronic kidney disease and the risks of death, cardiovascular events, and hospitalization. N Engl J Med 2004; 351(13): 1296-305.

[29] Anavekar NS, McMurray JJ, Velazquez EJ, et al. Relation between renal dysfunction and cardiovascular outcomes after myocardial infarction. N Engl J Med 2004; 351(13): 1285-95.

[30] Weiner DE, Tighiouart H, Amin MG, et al. Chronic kidney disease as a risk factor for cardiovascular disease and all-cause mortality: a pooled analysis of community based studies. J Am Soc Nephrol 2004; 15(5): 1307-15.

[31] Tonelli M, Klarenbach SW, Lloyd AM, et al. Higher estimated glomerular filtration rates may be associated with increased risk of adverse outcomes, especially with concomitant proteinuria. Kidney Int 2011; 80(12): 1306-14.

[32] Ohsawa M, Tanno K, Itai K, et al. Comparison of predictability of future cardiovascular events between chronic kidney disease (CKD) stage based on CKD Epidemiology Collaboration equation and that based onmodification of diet in renal disease equation in the Japanese general Population. Circ J 2013; 77(5): 1315-25.

[33] Matsushita K, Selvin E, Bash LD, et al. Risk implications of the new CKD Epidemiology Collaboration (CKD-EPI) equation compared with the MDRD study equation for estimated GFR: the Atherosclerosis Risk in Communities (ARIC) study. Am J Kidney Dis 2010; 55(4): 648-59. 
[34] Nagata $M$, Ninomiya $T$, Kiyohara $Y$, et al. Prediction of cardiovascular disease mortality by proteinuria and reduced kidney function: pooled analysis of 39,000 individuals from 7 cohort studies in Japan. Am J Epidemiol 2013; 178(1): 1-11.

[35] Tonelli M, Muntner P, Lloyd A, et al. Using proteinuria and estimated glomerular filtration rate to classify risk in patients with chronic kidney disease: a cohort study. Ann Intern Med 2011; 154(1): 12-21.

[36] Al Suwaidi J, Reddan DN, Williams K, et al. Prognostic implications of abnormalities in renal function in patients with acute coronary syndromes. Circulation 2002, 106(8): 974980.

[37] Damman K, Navis G, Voors AA, et al. Worsening renal function and prognosis in heart failure: systematic review and meta-analysis. J Card Fail 2007, 13(8): 599-608.
[38] Hillege HL, Nitsch D, Pfeffer MA, et al. Renal function as a predictor of outcome in a broad spectrum of patients with heart failure. Circulation 2006, 113(5): 671-678.

[39] Kim CS, Choi JS, Park JW, et al. Concomitant renal insufficiency and diabetes mellitus asprognostic factors for acute myocardial infarction. Cardiovasc Diabetol 2011, 10: 95 .

[40] Schrier RW. Role of diminished renal function in cardiovascular mortality: marker or pathogenetic factor? J Am Coll Cardiol 2006, 47(1): 1-8.

[41] Vanholder R, Massy Z, Argiles A, et al. Chronic kidney disease as cause of cardiovascular morbidity and mortality. Nephrol Dial Transplant 2005, 20(6): 1048-1056. 